\title{
Legendre transforms in chemical thermodynamics
}

\author{
Robert A. Alberty \\ Massachusetts Institute of Technology, Cambridge, MA 02139, USA \\ FAX:617-253-7030 email: alberty@mit.edu
}

\begin{abstract}
Legendre transforms are used to introduce intensive variables as natural variables in the fundamental equations of thermodynamics. Natural variables are important because when a thermodynamic potential can be determined as a function of its natural variables, all the other thermodynamic properties of the system can be obtained by taking partial derivatives. It is usually more convenient to use thermodynamic potentials that have intensive natural variables because they are often more easily controlled than the conjugate extensive variables. This is illustrated for chemical reaction systems in which it is of interest to specify the chemical potential of a species (for example, the $\mathrm{pH}$ in a biochemical system). Since the electric potentials of phases in a multi-phase system are not natural variables of the Gibbs energy, it is useful to define a transformed Gibbs energy, for which electric potentials of phases are natural variables. The use of a Legendre transform brings in a new set of thermodynamic properties, new Maxwell equations, Gibbs-Duhem equations, and Gibbs-Helmholtz equations.
\end{abstract}

\section{INTRODUCTION}

This paper is about the most fundamental concepts of thermodynamics for systems involving work in addition to $P V$ work. The fundamental equation for the internal energy involves the extensive natural variables $S$ and $V$ (ref. 1):

$\mathrm{d} U=T \mathrm{~d} S-P \mathrm{~d} V$

Natural variables are important because if a thermodynamic potential can be determined experimentally as a function of its natural variables, all of the other thermodynamic properties for the system can be calculated by taking partial derivatives. In equation 1 there are two pairs of conjugate variables $(T, S)$ and $(P, V)$. The thermodynamic potentials enthalpy $H$, Helmholtz energy $A$, and Gibbs energy $G$ are introduced by use of the Legendre transforms

$$
\begin{aligned}
& H=U+P V \\
& A=U-T S \\
& G=U+P V-T S
\end{aligned}
$$

A Legendre transform is a change in natural variables that is accomplished by defining a new thermodynamic potential by subtracting from the internal energy (or other thermodynamic potential) one or more products of conjugate variables. The result of a Legendre transform is the introduction of one or more intensive natural variables into the fundamental equation, instead of their conjugate extensive variables. A Legendre transform seems like such a simple thing, but it has a very big effect on the convenience in making thermodynamic calculations. The four thermodynamic potentials in equations 1-4 are useful because they can be used to express criteria for spontaneous change and equilibrium for various choices of natural variables: $(\mathrm{d} U)_{S, V} \leq 0,(\mathrm{~d} H)_{S, P} \leq 0,(\mathrm{~d} A)_{T, V} \leq 0$, and $(\mathrm{d} G)_{T, P} \leq 0$, as indicated by the subscripts, which indicate the natural variables that are held constant. The thermodynamic potentials $U$, $H, A$, and $G$ for a system can only decrease when their natural variables are held constant, and their differentials for differential changes at equilibrium are equal to zero.

The use of Legendre transforms is not restricted to thermodynamics. A Legendre transform has made a major contribution in mechanics. The Lagrangian function $L$ is a function of coordinates and velocities, but it is often more convenient to define the Hamiltonian function $H$ with a Legendre transform because the Hamiltonian is a function of coordinates and momenta. Quantum mechanics is based on the Hamiltonian rather than the Lagrangian function.

A closed system involving $P V$ work involves two conjugate pairs of extensive and intensive variables, and this leads to four thermodynamic potentials. This can be generalized because the number of thermodynamic potentials for a given system is equal to $2^{k}$, where $k$ is the number of independent conjugate pairs. As indicated by equation 1, the basic theory of thermodynamics is based on calculus, and so thermodynamics involves an interconnected set of equations: fundamental equations, integrated fundamental equations (Euler equations), partial derivatives of thermodynamic potentials, Maxwell 
equations, Gibbs-Helmholtz equations, and Gibbs-Duhem equations that come from the operations of calculus. Gibbs-Duhem equations can be regarded as complete Legendre transforms, in that all products of conjugate variables are subtracted from the internal energy. It is important to notice that intensive variables become natural variables only when they are introduced by means of Legendre transforms of the internal energy.

When non- $P V$ work is involved in a thermodynamic system, $2^{k}$ can be much larger than 4 and the larger number of thermodynamic potentials increases nomenclature problems because each new thermodynamic potential that is defined by a Legendre transform needs a symbol and a name. Callen discussed this problem in his book Thermodynamics and An Introduction to Thermostatistics (ref. 2) and recommended a nomenclature that can represent all thermodynamic potentials that can be defined. He suggested the use of square brackets to indicate the intensive variables that have been introduced through Legendre transforms of the internal energy. For example, Callen symbols for the thermodynamic potentials introduced by Legendre transforms 2-4 are $H=U[P], A=U[T]$, and $G=U[T, P]$. Callen's symbols are not used in this article, but they show that it is possible to give a symbol for any thermodynamic potential. Natural variables must be independent, and so we must consider the independence of both intensive and extensive variables.

This paper is concerned with chemical work and work of electric transport, but it is clear that these same considerations apply to gravitational work, centrifugal work, mechanical work on solids, surface work, work of electric polarization, and work of magnetic polarization.

\section{LEGENDRE TRANSFORM TO INTRODUCE THE CHEMICAL POTENTIAL OF A SPECIES}

When chemical work is involved in a single-phase system, the fundamental equation for $G$ becomes $N$

$$
\mathrm{d} G=-S \mathrm{~d} T+V \mathrm{~d} P+\sum \mu_{i} \mathrm{~d} n_{i}
$$

where $N$ is the number of species. When there are no chemical reactions, the amounts of species are natural variables, and the criterion of equilibrium based on $\mathrm{G}$ is $(\mathrm{d} G)_{T, P,\left\{n_{i}\right\}} \leq 0$, where $\left\{n_{i}\right\}$ is the set of amounts of species. But when there are chemical reactions, the amounts of species are not independent variables and cannot be natural variables. At chemical equilibrium, $\Sigma v_{i} \mu_{i}=0$ for each of the $R$ independent reactions for the system, where the $v_{i}$ are stoichiometric numbers that are positive for products and negative for reactants. These $R$ relations can be used to eliminate $R$ chemical potentials of species from equation 5 to obtain

$$
\mathrm{d} G=-S \mathrm{~d} T+V \mathrm{~d} P+\sum_{i=1} \mu_{i} \mathrm{~d} n_{i}^{\prime}
$$

where $n_{i}$ ' is the amount of component $i$. The number $C$ of components is equal to the number $N$ of species minus the number $R$ of independent reactions; $C=N-R$ (ref. 3). Components are independent variables because components are conserved when reactions occur. The number of components is often equal to the number of elements, but that is not necessarily true. If two elements in a system always occur in the same ratio, as in $\mathrm{SO}_{4}$, the combination counts as an element, and the number of components is reduced by one. There may be additional components, like electric charge, but this constraint is not necessarily independent of element balances. Further constraints may be introduced by mechanisms that cause the number of reactions that actually occur to be less than the number of possible independent reactions. The determination of the number of constraints of complex systems really requires computers with mathematical programs because the number of components is equal to the rank of the conservation matrix for the system (ref. 3). The choice of components is not unique, but the number of components is unique. Legendre transforms can be used to introduce up to $C-1$ chemical potentials of species as natural variables.

My own introduction to the possibility of using the chemical potential of a species as a natural variable was not in terms of Legendre transforms, but was more down to earth. When I was working on the thermodynamics of petroleum processing, I learned about calculations at Mobil Research and Development that gave me an idea. They were making equilibrium calculations on a reactor, which for some reason had a nearly constant partial pressure of molecular hydrogen, let's say $10^{-3}$ bar. To simplify the calculations, which involved a very large number of species, someone suggested that since the standard state pressure for molecular hydrogen of $1 \mathrm{bar}$ is arbitrary, they might as well redefine the standard state of molecular hydrogen to be $10^{-3}$ bar and then leave molecular hydrogen out of the calculations. They did this by adjusting the standard Gibbs energies of formation of the various species in the system according to their hydrogen content. At that time I was making calculations on the degrees of polymerization in homologous 
series in complex mixtures of hydrocarbons under various conditions, and I recognized that I could set the partial pressure of ethylene at some specified value and recalculate the standard Gibbs energies of formation for the other species using ethylene at a specified partial pressure as a reference state. After doing this for some time and working with Irwin Oppenheim on the statistical mechanics of these systems (ref. 4-6), we realized that we were really using a Legendre transform to define a new thermodynamic potential, which has its minimum value at specified $T, P$, and $P\left(\mathrm{C}_{2} \mathrm{H}_{4}\right)$.

As a simple example of a Legendre transform of the Gibbs energy for a system that involves chemical work, consider a chemical reaction system containing species $\mathrm{A}, \mathrm{B}$, and $\mathrm{C}$ that react according to

$$
A+B=C
$$

The differential of the Gibbs energy at constant $T$ and $P$ is given by

$$
(\mathrm{d} G)_{T, P}=\mu_{\mathrm{A}} \mathrm{d} n_{\mathrm{A}}+\mu_{\mathrm{B}} \mathrm{d} n_{\mathrm{B}}+\mu_{\mathrm{C}} \mathrm{d} n_{\mathrm{C}}
$$

When reaction 7 is at equilibrium, the equilibrium relation

$$
\mu_{\mathrm{A}}+\mu_{\mathrm{B}}=\mu_{\mathrm{C}}
$$

can be used to eliminate $\mu_{\mathrm{C}}$ from equation 8 to obtain

$$
(\mathrm{d} G)_{T, P}=\mu_{\mathrm{A}}\left(\mathrm{d} n_{\mathrm{A}}+\mathrm{d} n_{\mathrm{C}}\right)+\mu_{\mathrm{B}}\left(\mathrm{d} n_{\mathrm{B}}+\mathrm{d} n_{\mathrm{C}}\right)=\mu_{\mathrm{A}} \mathrm{d} n_{\mathrm{A}}{ }^{\prime}+\mu_{\mathrm{B}} \mathrm{d} n_{\mathrm{B}}{ }^{\prime}
$$

where $n_{A^{\prime}}=n_{\mathrm{A}}+n_{\mathrm{C}}$ is the amount of component $\mathrm{A}$ and $n_{\mathrm{B}^{\prime}}=n_{\mathrm{B}}+n_{\mathrm{C}}$ is the amount of component $\mathrm{B}$. Note that there are two components because $C=N-R=3-1=2$. The amounts of the two components, $n_{\mathrm{A}}{ }^{\prime}$ and $n_{\mathrm{B}}{ }^{\prime}$, are independent variables, in contrast with the amounts of species, $n_{\mathrm{A}}, n_{\mathrm{B}}$, and $n_{\mathrm{C}}$. The fact that components have the same chemical potentials as species is not often discussed in the literature, but Beattie and Oppenheim discuss it in their book Principles of Thermodynamics (ref. 7).

Now, let us think about reaction 7 being carried out in a reactor that is connected to a reservoir for $\mathrm{B}$ with a semipermeable membrane that is permeable to species $B$. The pressure of $B$ in the reservoir can be maintained at any desired value. Since we want to make the chemical potential of $B$ a natural variable, we use the following Legendre transform to define a transformed Gibbs energy $G^{\prime}$ :

$$
G^{\prime}=G-n_{\mathrm{B}} \mu_{\mathrm{B}}
$$

Note that the conjugate extensive variable of the chemical potential of a species is not the amount $n_{\mathrm{B}}$ of the species in a chemically reacting system because $n_{\mathrm{B}}$ is not an independent variable. The amount $n_{\mathrm{B}}$ ' of component $B$ is an independent variable. The amount $n_{B}$ ' of component $B$ is the amount of free $B$ plus the amount of bound $\mathrm{B}$, namely $\mathrm{C}$, in the reactor. Taking the differential of $G^{\prime}$ and substituting $(\mathrm{d} G)_{T, P}$ from equation 10 yields

$$
\left(\mathrm{d} G^{\prime}\right)_{T, P}=\mu_{\mathrm{A}} \mathrm{d} n_{\mathrm{A}}{ }^{\prime}-n_{\mathrm{B}}{ }^{\prime} \mathrm{d} \mu_{\mathrm{B}}
$$

When the chemical potential of $B$ is specified,

$$
\left(\mathrm{d} G^{\prime}\right)_{T, P, \mu_{\mathrm{B}}}=\mu_{\mathrm{A}} \mathrm{d} n_{\mathrm{A}}{ }^{\prime}
$$

and the system becomes effectively a one-component system. The number $C^{\prime}$ of apparent components is equal to the number $N^{\prime}$ of species with variable concentrations minus the number $R^{\prime}$ of independent reactions; $C^{\prime}=N^{\prime}-R^{\prime}=2-1=1$. According to Callen's suggestion $G^{\prime}$ could be represented by $U\left[T, P, \mu_{\mathrm{B}}\right]$, but the advantage of the symbol $G^{\prime}$ is that this thermodynamic potential is very much like $G$ because the criterion for equilibrium becomes $\left(\mathrm{d} G^{\prime}\right)_{T, P, \mu_{\mathrm{B}}} \leq 0$. For ideal gases, $\left(\mathrm{d} G^{\prime}\right)_{T, P, P_{\mathrm{B}}} \leq 0$.

Making the Legendre transformation in equation 11 has had the effect of making it possible to describe this chemical equilibrium in a simpler way. We have gone from three species at $T, P$ to one component at $T$, $P, \mu_{\mathrm{B}}$. This may not seem very important for such a simple system, but it is very useful for complex systems. Since $\mathrm{d} n_{\mathrm{A}}{ }^{\prime}=\mathrm{d} n_{\mathrm{A}}+\mathrm{d} n_{\mathrm{C}}$ and $\mu_{\mathrm{A}}=\mu_{\mathrm{C}}-\mu_{\mathrm{B}}$, equation 13 can be written

$$
\left(\mathrm{d} G^{\prime}\right)_{T, P, \mu_{\mathrm{B}}}=\mu_{\mathrm{A}} \mathrm{d} n_{\mathrm{A}^{\prime}}=\mu_{\mathrm{A}}\left(\mathrm{d} n_{\mathrm{A}}+\mathrm{d} n_{\mathrm{C}}\right)=\mu_{\mathrm{A}} \mathrm{d} n_{\mathrm{A}}+\left(\mu_{\mathrm{C}}-\mu_{\mathrm{B}}\right) \mathrm{d} n_{\mathrm{C}}
$$

Equation 14 has two partial derivatives that define transformed chemical potentials $\mu_{i}^{\prime}$ for $\mathrm{A}$ and $\mathrm{C}$ :

$$
\begin{aligned}
& \left(\frac{\partial G^{\prime}}{\partial n_{\mathrm{A}}}\right)_{T, P, n_{\mathrm{C},} \mu_{\mathrm{B}}}=\mu_{\mathrm{A}^{\prime}}=\mu_{\mathrm{A}} \\
& \left(\frac{\partial G^{\prime}}{\partial n_{\mathrm{C}}}\right)_{T, P, n_{\mathrm{A}}, \mu_{\mathrm{B}}}=\mu_{\mathrm{C}^{\prime}}=\mu_{\mathrm{C}}-\mu_{\mathrm{B}}
\end{aligned}
$$

The transformed chemical potential of species $\mathrm{A}$ is equal to its chemical potential $\mu_{\mathrm{A}}$, but the transformed chemical potential of species $C$ is equal to $\mu_{C}-\mu_{B}$.

Equation 14 can be rewritten in terms of these transformed chemical potentials.

$$
\left(\mathrm{d} G^{\prime}\right)_{T, P, \mu_{\mathrm{B}}}=\mu_{\mathrm{A}}{ }^{\prime} \mathrm{d} n_{\mathrm{A}}+\mu_{\mathrm{C}} \mathrm{d} n_{\mathrm{C}}
$$


Thus when the chemical potential of a species is specified, the transformed chemical potentials $\mu_{i}^{\prime}$ of the variable species ( $A$ and $C$ ) replace their chemical potentials in the fundamental equation. Equation 17 can be used to show that at equilibrium at specified $\mu_{B}$ the transformed chemical potentials of $A$ and $C$ are equal, $\mu_{\mathrm{A}}{ }^{\prime}=\mu_{\mathrm{C}^{\prime}}$. Equation 17 is like the fundamental equation for $G$ for two isomers. At chemical equilibrium, the chemical potentials of isomers are equal. An isomer group at equilibrium has a certain standard Gibbs energy of formation and standard enthalpy of formation (ref. 8). Thus we can interpret equation 17 by saying that at equilibrium, pseudoisomer $A$ has the same transformed chemical potential as pseudoisomer $\mathrm{C}$. Since the pseudoisomer group A,C has a transformed chemical potential $\mu_{\mathrm{A}}{ }^{\prime}$ and the amount of the pseudoisomer group is represented by $n_{\mathrm{A}^{\prime}}$, equation 13 can be written

$$
\left(\mathrm{d} G^{\prime}\right) T, P, \mu_{\mathrm{B}}=\mu_{\mathrm{A}}{ }^{\prime} \mathrm{d} n_{\mathrm{A}}{ }^{\prime}
$$

This equation can be integrated at constant $T, P, \mu_{\mathrm{B}}$ to obtain the Euler equation $G^{\prime}=n_{\mathrm{A}}{ }^{\prime} \mu_{\mathrm{A}^{\prime}}$.

If there are more species and more pseudoisomer groups, equation 18 can be generalized to

$$
\left(\mathrm{d} G^{\prime}\right)_{T, P, \mu_{\mathrm{B}}}=\sum_{i=1}^{N} \mu_{i}^{\prime} \mathrm{d} n_{i}{ }^{\prime}
$$

where the $\mu_{\mathrm{i}}^{\prime}$ are the transformed chemical potentials of the $N$ pseudoisomer groups and the $n_{i}{ }^{\prime}$ are the amounts of the $N^{\prime}$ pseudoisomer groups. This equation looks very much like equation 8 for $(\mathrm{dG})_{\mathrm{T}, \mathrm{P}}$ except that it is for the transformed Gibbs energy and is written in terms of pseudoisomer groups at specified $\mu_{\mathrm{B}}$, rather than species. The fact that equation 19 looks so much like equation 8 suggests that the pseudoisomer groups can be treated like species are treated in familiar chemical equilibrium calculations. The fundamental equation for $\mathrm{G}^{\prime}$ yields new Maxwell equations, a Gibbs-Duhem equation, and a GibbsHelmholtz equation. Equation 19 can be integrated at constant $T, P, \mu_{\mathrm{B}}$ to obtain the Euler equation

$$
G^{\prime}=\sum_{i=1}^{N^{\prime}} \mu_{i}^{\prime} n_{i}^{\prime}
$$

Thus the transformed Gibbs energy $G^{\prime}$ is additive in the transformed chemical potentials $\mu_{i}^{\prime}$ of the pseudoisomer groups, just like the Gibbs energy $G$ is additive in the chemical potentials $\mu_{i}$ of species. When the chemical potential of a species is specified, the thermodynamic treatment of the reaction system can be reconceptualized in terms of pseudoisomer groups. A pseudoisomer group is a sum of species differing only with respect to the number of $B$ molecules in the species.

Equation 20 can be derived in another way that is useful because it is more general and provides a relation for the calculation of the transformed chemical potential of a species. We start with the Legendre transform in equation 11 and substitute $G=\Sigma \mu_{i} n_{i}$ and $n_{\mathrm{B}}{ }^{\prime}=\Sigma N_{\mathrm{B}}(i) n_{i}$, where $N_{\mathrm{B}}(\mathrm{i})$ is the number of B molecules in a molecule of $i$, to obtain

$$
G^{\prime}=\sum_{i=1}^{N} \mu_{i} n_{i}-\mu_{\mathrm{B}} \sum_{i=1}^{N} N_{\mathrm{B}}(i) n_{i}=\sum_{i=1} n_{i}\left[\mu_{i}-\mu_{\mathrm{B}} N_{\mathrm{B}}(i)\right]=\sum_{i=1} \sum_{i} \mu_{i}^{\prime}=\sum_{i=1} n_{i}{ }^{\prime} \mu_{i}^{\prime}
$$

where the transformed chemical potential of $i$ is given by

$$
\mu_{i}^{\prime}=\mu_{i}-N_{\mathrm{B}}(i) \mu_{\mathrm{B}}
$$

Derivations are carried out with chemical potentials, but calculations on actual systems are carried out with standard Gibbs energies of formation, so that equation 22 can be written as

$$
\Delta_{\mathrm{f}} G_{i}{ }^{\circ}=\Delta_{\mathrm{f}} G_{i}{ }^{\circ}-N_{\mathrm{B}}(i)\left[\Delta_{\mathrm{f}} G_{\mathrm{B}}{ }^{\mathrm{o}}+R T \ln \left(P_{\mathrm{B}} / P^{\circ}\right)\right]
$$

where the gases are assumed to be ideal.

Just as isomer groups can be treated as species in equilibrium calculations at specified $T$ and $P$ (ref. 8), pseudoisomer groups at a specified chemical potential of a species can be treated as pseudo "species." The standard transformed Gibbs energy of formation of a pseudoisomer group is given by

$$
\Delta_{\mathrm{f}} G^{\prime} \text { (pseudoisomer group) }=-R T \ln \left\{\sum_{i=1} \exp \left[-\Delta_{\mathrm{f}} G_{i}^{\prime} / R T\right]\right\}
$$

The mole fraction $r_{i}$ of the $i$ th pseudoisomer in the pseudoisomer group is given by

$$
r_{i}=\exp \left\{\left[\Delta_{\mathrm{f}} G^{\prime} \mathrm{o} \text { (pseudoisomer group) }-\Delta_{\mathrm{f}} G_{i}{ }^{\circ}\right] / R T\right\}
$$


The summation in equation 24 is a kind of partition function, and the distribution in equation 25 is a kind of Boltzmann distribution. The standard transformed enthalpy of formation of the pseudoisomer group is a mole fraction weighted average and is given by

$$
\Delta_{\mathrm{f}} H^{\prime \circ} \text { (pseudoisomer group) }=\sum_{i=1}^{N_{i}} r_{\mathrm{f}} H_{i}{ }^{\circ}
$$

There is a corresponding standard transformed entropy of formation. Thus the use of Legendre transform brings in a whole set of transformed thermodynamic properties.

This approach has been used to calculate equilibrium distributions in a number of hydrocarbon homologous series as functions of $P\left(\mathrm{C}_{2} \mathrm{H}_{4}\right), P\left(\mathrm{C}_{2} \mathrm{H}_{2}\right), P\left(\mathrm{H}_{2}\right)$, and $P(\mathrm{H})($ ref. 9).

\section{LEGENDRE TRANSFORM TO INTRODUCE pH AND pMg AS NATURAL
VARIABLES}

Biochemistry provides a good example of the usefulness of the transformed Gibbs energy because biochemists are primarily interested in what happens at a specified $\mathrm{pH}$. Since most biochemical reactants are weak acids, specifying the chemical potential of $\mathrm{H}^{+}$means that the Gibbs energy is not the criterion of equilibrium. To make the chemical potential of $\mathrm{H}^{+}$a natural variable, the following Legendre transform is used (ref. 10-11):

$$
G^{\prime}=G-n^{\prime}\left(\mathrm{H}^{+}\right) \mu\left(\mathrm{H}^{+}\right)
$$

where $n^{\prime}\left(\mathrm{H}^{+}\right)$is the total amount of hydrogen in the system, namely the amount of the hydrogen component, which is given by

$$
n^{\prime}\left(\mathrm{H}^{+}\right)=\sum_{i=1} N_{\mathrm{H}}(i) n_{i}
$$

where $N_{\mathrm{H}}(i)$ is the number of hydrogen atoms in species $i$, and $n_{i}$ is the amount of species $i$. Substituting this and $\mathrm{G}=\Sigma \mu_{i} n_{i}$ into the Legendre transform (equation 27) yields

$$
\begin{aligned}
G^{\prime} & =\sum n_{i} \mu_{i}-\sum N_{\mathrm{H}}(i) \mu\left(\mathrm{H}^{+}\right) n_{i} \\
& =\sum_{n_{i}}\left[\mu_{i}-N_{\mathrm{H}}(i) \mu\left(\mathrm{H}^{+}\right)\right] \\
& =\sum_{n_{i} \mu_{i}}
\end{aligned}
$$

where the transformed chemical potential $\mu_{i}$ of species $i$ is given by

$$
\mu_{i}^{\prime}=\mu_{i}-N_{\mathrm{H}}(i) \mu\left(\mathrm{H}^{+}\right)
$$

Substituting this equation in equation 5 yields

$$
\mathrm{d} G=-S \mathrm{~d} T+V \mathrm{~d} P+\sum_{i=1}^{N-1} \mu_{i}^{\prime} \mathrm{d} n_{i}+\mu\left(\mathrm{H}^{+}\right) \sum_{i=1}^{N} N_{\mathrm{H}}(i) \mathrm{d} n_{i}
$$

Since the amount $n^{\prime}\left(\mathrm{H}^{+}\right)$of the hydrogen component is given by equation 28 , equation 31 can be written $N-1$

$$
\mathrm{d} G=-S \mathrm{~d} T+V \mathrm{~d} P+\sum_{i=1} \mu_{i}{ }^{\prime} \mathrm{d} n_{i}+\mu\left(\mathrm{H}^{+}\right) \mathrm{d} n^{\prime}\left(\mathrm{H}^{+}\right)
$$

Since the hydrogen component contributes a single term, we can use the Legendre transform to interchange the roles of the intensive $\left(\mu\left(\mathrm{H}^{+}\right)\right)$and extensive $\left(n^{\prime}\left(\mathrm{H}^{+}\right)\right)$variables in the last term.

The differential of $G^{\prime}$ (equation 27 ) is

$$
\mathrm{d} G^{\prime}=\mathrm{d} G-n^{\prime}\left(\mathrm{H}^{+}\right) \mathrm{d} \mu\left(\mathrm{H}^{+}\right)-\mu\left(\mathrm{H}^{+}\right) \mathrm{d} n^{\prime}\left(\mathrm{H}^{+}\right)
$$

and substituting equation 32 yields

$$
\mathrm{d} G^{\prime}=-S \mathrm{~d} T+V \mathrm{~d} P+\sum_{i=1}^{N-1} \mu_{i}^{\prime} \mathrm{d} n_{i}-n^{\prime}\left(\mathrm{H}^{+}\right) \mathrm{d} \mu\left(\mathrm{H}^{+}\right)
$$

We can make the hydrogen ion concentration the independent variable, rather than $\mu\left(\mathrm{H}^{+}\right)$, by use of 


$$
\mathrm{d} \mu\left(\mathrm{H}^{+}\right)=\left\{\frac{\partial \mu\left(\mathrm{H}^{+}\right)}{\partial T}\right\}_{\left[\mathrm{H}^{+}\right]} \mathrm{d} T+\left\{\frac{\partial \mu\left(\mathrm{H}^{+}\right)}{\partial\left[\mathrm{H}^{+}\right]}\right\}_{T} \mathrm{~d}\left[\mathrm{H}^{+}\right]
$$

When this equation is substituted in equation 34 and we take the indicated derivatives, we obtain

$$
\mathrm{d} G^{\prime}=-S \mathrm{~d} T+V \mathrm{~d} P+\sum_{i=1}^{N-1} \mu_{i}^{\prime} \mathrm{d} n_{i}+2.303 n^{\prime}\left(\mathrm{H}^{+}\right) R T \mathrm{dpH}
$$

where $\mathrm{pH}=-\log \left(\left[\mathrm{H}^{+}\right] / \mathrm{c}^{0}\right)$ and $\mathrm{c}^{0}$ is the standard concentration. The transformed entropy $S^{\prime}$ of the system is given by

$$
S^{\prime}=S-n^{\prime}\left(\mathrm{H}^{+}\right) \bar{S}\left(\mathrm{H}^{+}\right)
$$

where $\bar{S}\left(\mathrm{H}^{+}\right)$is the molar entropy of hydrogen ion. Defining the transformed enthalpy as $H^{\prime}=G^{\prime}+T S^{\prime}$ yields

$$
H^{\prime}=H \cdot n^{\prime}\left(\mathrm{H}^{+}\right) \bar{H}\left(\mathrm{H}^{+}\right)
$$

Equation 36 shows that the criterion for spontaneous change and equilibrium is ( $\left.\mathrm{d} G{ }^{\prime}\right)_{T, P, \mathrm{pH}} \leq 0$. Thus the transformed Gibbs energy $G^{\prime}$ is minimized at equilibrium at specified $T, P$, and $\mathrm{pH}$.

Since $\mathrm{Mg}^{2+}$ is often bound by biochemical reactants, the chemical potential of $\mathrm{Mg}^{2+}$ can also be included in the Legendre transform given in equation 27. When this is done equation 36 can be written

$$
\left(\mathrm{d} G^{\prime}\right)_{\mathrm{pH}, \mathrm{pMg}}=-S^{\prime} \mathrm{d} T+V \mathrm{~d} P+\sum_{i=1} \mu_{i}^{\prime} \mathrm{d} n_{i}
$$

where the transformed chemical potential of species $i$ is given by

$$
\mu_{i}^{\prime}=\mu_{i}-N_{\mathrm{H}}(i) \mu\left(\mathrm{H}^{+}\right)+N_{\mathrm{Mg}}(i) \mu\left(\mathrm{Mg}^{2+}\right)
$$

To see how this applies to a biochemical reaction system, consider adenosine triphosphate (ATP), which is the sum of ATP4-, HATP ${ }^{3-}, \mathrm{H}_{2} \mathrm{ATP}^{2-}, \mathrm{MgATP}^{2-}, \mathrm{MgHATP}-$, and $\mathrm{Mg}_{2} \mathrm{ATP}$. At specified $\mathrm{pH}$ and $\mathrm{pMg}$, these six species are pseudoisomers and have the same transformed chemical potential $\mu_{i}^{\prime}$. Therefore, equation 39 for a biochemical reaction system can be written in terms of the differential amounts $\mathrm{d} n_{i}$ ' of pseudoisomer groups, rather than species, to obtain

$$
\left(\mathrm{d} G^{\prime}\right)_{\mathrm{pH}, \mathrm{pMg}}=-S^{\prime} \mathrm{d} T+V \mathrm{~d} P+\sum_{i=1}^{N} \mu_{i}{ }^{\prime} \mathrm{d} n_{i}{ }^{\prime}
$$

where $N$ is the number of pseudoisomer groups.

Equation 41 can be used to derive the expression for the apparent equilibrium constant $K^{\prime}$, which is written in terms of reactants (sums of species). The values of $K^{\prime}$ for a number of biochemical reactions and their temperature coefficients, or calorimetric data, can be used to calculate standard transformed Gibbs energies and standard transformed enthalpies of reaction of the reactants by using

$$
\begin{aligned}
\Delta_{\mathrm{r}} G^{\prime \circ}= & -R T \ln K^{\prime}=\sum_{i=1}^{N^{\prime}} v_{i} \Delta_{\mathrm{f}} G_{i}{ }^{\prime} \mathrm{O} \\
\Delta_{\mathrm{r}} H^{\prime \circ} \mathrm{O} & \sum_{i=1}^{\prime} v_{i} \Delta_{\mathrm{f}} H_{i}^{\prime \circ}
\end{aligned}
$$

where the $v_{i}^{\prime}$ are stoichiometric numbers for the reactants. Biochemical reactions are written in terms of sums of species at specified $\mathrm{pH}$ and $\mathrm{pMg}$ and should not include $\mathrm{H}^{+}$or $\mathrm{Mg}^{2+}$ because they are not conserved. In order to discuss metabolism, biochemists need a different kind of thermodynamic table than chemists because they are interested in reactants, like ATP, and are not interested in dealing with all of the species that make up ATP. Thus they are interested in the transformed thermodynamic properties of sums of species at a particular $\mathrm{pH}$ value, and perhaps a specified free concentration of metal ions, like $\mathrm{Mg}^{2+}$, that are bound by the reactant species. 
By use of the equations discussed here, thermodynamic tables can be prepared at specified $\mathrm{pH}$ and $\mathrm{pMg}$, for example, when the $\Delta_{\mathrm{f}} G^{\circ}$ and $\Delta_{\mathrm{f}} H^{\circ}$ values of the various species are known (ref. 12-14). There is a problem in calculating $\Delta_{\mathrm{f}} G^{\circ}$ and $\Delta_{\mathrm{f}} H^{\circ}$ for the more complicated molecules of biochemical interest, even when the acid dissociation and metal-complex dissociation constants are known. The problem is that it is not yet possible to relate the thermodynamic properties of these complicated molecules to the elements in their reference states. However, this problem can be handled by assigning one species $\Delta_{\mathrm{f}} G^{0}=\Delta_{\mathrm{f}} H^{0}=0$ and stating this as a convention of the table. In the absence of data on $\Delta_{\mathrm{f}} G^{\circ}$ and $\Delta_{\mathrm{f}} H^{\circ}$ for species, $\Delta_{\mathrm{f}} G^{\prime o}$ and $\Delta_{\mathrm{f}} H^{\circ}$ can be calculated from $K^{\prime}$ values and heats of reaction (ref. 15) at specified $\mathrm{pH}$ and $\mathrm{pMg}$ by using equations 42-43. This nomenclature has been approved by IUPAC and IUBMB (ref. 16).

I like to think of equilibrium calculations at specified $\mathrm{pH}$ as being at Level 2. Level 1 equilibrium calculations are the familiar calculations in terms of species. The advantage of Level 2 is that it deals with sums of species, like ATP, and so there is a simplification in the description of the system. Calculations on biochemical systems can also be carried out at Level 3 (ref. 17). As an illustration of Level 3, consider the glucokinase reaction:

ATP + Glucose = ADP + Glucose 6-phosphate

ATP + Glucose $=\mathrm{ADP}+\mathrm{Glucose} 6$-phosphate
The apparent equilibrium constant $K^{\prime}$ for this reaction at specified $T, P, \mathrm{pH}, \mathrm{pMg}$, and ionic strength is given by

$$
K^{\prime}=\frac{[\text { ADP }][\text { Glucose 6-phosphate }]}{[\text { ATP][Glucose] }}
$$

In considering systems of reactions like this, biochemists are often interested in the extent to which glucose is phosphorylated at equilibrium at specified concentrations of ATP and ADP because these reactants are involved in many reactions in a living cell and may have rather steady concentrations. Equilibrium calculations at specified $T, P, \mathrm{pH}, \mathrm{pMg},[\mathrm{ATP}]$, and $[\mathrm{ADP}]$ are Level 3 calculations. To make [ATP] and [ADP] natural variables it is necessary to define a further transformed Gibbs energy $G^{\prime \prime}$ with the following Legendre transform:

$$
G^{\prime \prime}=G^{\prime}-n^{\prime}(\mathrm{ATP}) \mu^{\prime}(\mathrm{ATP})-n^{\prime}(\mathrm{ADP}) \mu^{\prime}(\mathrm{ADP})
$$

There are two reactants (glucose and glucose 6-phosphate) and one component, and so glucose and glucose- 6 phosphate are pseudoisomers under these conditions. Since $R^{\prime \prime}=N^{\prime \prime}-C^{\prime \prime}=2-1=1$, there is a single apparent reaction at specified $\mathrm{T}, \mathrm{P}, \mathrm{pH},[\mathrm{ATP}]$, and [ADP]:

Glucose = Glucose 6-phosphate

The fundamental equation is now

$$
\left(\mathrm{d} G^{\prime \prime}\right)_{T, P, \mathrm{pH},} \text { [ATP],[ADP] }=\mu^{\prime \prime}(\mathrm{Glu}) \mathrm{d} n^{\prime \prime}(\mathrm{Glu})+\mu^{\prime \prime}(\mathrm{G} 6 \mathrm{P}) \mathrm{d} n^{\prime \prime}(\mathrm{G} 6 \mathrm{P})
$$

and the apparent equilibrium constant of reaction 47 is defined by

$K^{\prime \prime}=$ [Glucose 6-phosphate]/[Glucose]

and can be calculated using the further transformed $\Delta_{\mathrm{f}} G^{\prime \prime} \mathrm{O}$ for glucose and glucose 6-phosphate. The advantage of the successive Legendre transforms is in the reduction in the number of equilibrium concentrations to be calculated for reaction 44 from 15 (Level 1), to 4 (Level 2), to 2 (Level 3).

Since the fundamental equations at the three levels are all of the same form, (1) expressions for $K, K^{\prime}$, and $K^{\prime \prime}$ are derived in the same way, (2) $S, S^{\prime}$, and $S^{\prime \prime}$ and $H, H$, and $H^{\prime \prime}$ are calculated in the same way, and (3) the same computer program (equcalc, ref. 18) can be used to calculate the equilibrium composition at each level. The equilibrium composition of a multi-reaction system can be calculated with a computer program that uses an iterative method. Fred Krambeck of Mobil Research and Development has written such a program, called equcalc, in $A P L$ and in Mathematica. This program, which is only about 20 lines long, uses the conservation matrix for the reaction system, calculates an initial estimate of the equilibrium composition, and then uses the Newton-Raphson method to minimize the Gibbs energy subject to the conservation conditions. Gas reactions and solution reactions have to be handled in slightly different ways because gas reactions are discussed in terms of partial pressures at a specified total pressure and solution reactions are discussed in terms of concentrations in a constant volume. The modified version of equcalc for solution reactions is referred to as equcalcc. Variations of these two basic programs are needed when the input is the stoichiometric number matrix rather than the conservation matrix. When the partial pressures or concentrations of one or more reactants are specified (Level 2), the same program can be used to calculate the equilibrium composition by minimizing the transformed Gibbs energy $G^{\prime}$, but with the specified component omitted from the conservation equations. The same program can be used at Level 3 to calculate the equilibrium composition that minimizes the further transformed Gibbs energy $G^{\prime \prime}$.

Legendre transforms can also be used in considering mechanical work, surface work, electrical work, work of electric polarization, and work of magnetic polarization (ref. 19), but there is only time to discuss one more example. 


\section{LEGENDRE TRANSFORM TO INTRODUCE ELECTRIC POTENTIALS OF PHASES AS NATURAL VARIABLES}

When a multi-phase system involves work of electric transport, terms of the form $\phi_{i} \mathrm{~d} Q_{i}=F \phi_{i} z_{i} \mathrm{~d} n_{i}$ are added to the fundamental equations for $U, H, A$, and $G$, where $\phi_{i}$ is the electric potential of the phase containing species $i, Q_{i}$ is the electric charge of species $i, F$ is the Faraday constant, and $z_{i}$ is the charge number of species $i$. Since the extensive variables in the electric work terms are $\mathrm{d} n_{i}$, the electric work terms are not independent of the chemical work terms, which also involve $\mathrm{d} n_{i}$. Since the electric work terms do not introduce new natural variables, the Gibbs energy $G$ continues to be the thermodynamic potential that provides the criterion of equilibrium at specified $T$ and $P$, and equation 5 applies. The electric potentials of the phases are constants for a particular system and need to be specified, but they are not natural variables of the Gibbs energy $G$. In order to make the electric potentials of the phases natural variables, it is necessary to make the following Legendre transform (ref. 20):

$$
G^{\prime}=G-\sum_{i=1}^{N} \phi_{i} Q_{i}=G-F \sum_{i=1}^{N} z_{i} \phi_{i} n_{i}
$$

where $N$ can be as large as the number of different species times the number of phases. Substituting $G=$ $\Sigma \mu_{i} n_{i}$ yields

$$
G^{\prime}=\sum_{i=1}^{N} \mu_{i} n_{i}-F \sum_{i=1}^{N} \sum_{i} \phi_{i} n_{i}=\sum_{i=1}^{N}\left(\mu_{i}-F z_{i} \phi_{i}\right) n_{i}=\sum_{i=1}^{N} \mu_{i}^{\prime} n_{i}
$$

where the transformed chemical potential $\mu_{i}^{\prime}$ of species $i$ is defined by

$$
\mu_{i}^{\prime}=\mu_{i}-F z_{i} \phi_{i}
$$

Equation 51 shows that the transformed Gibbs energy is additive in the transformed chemical potentials of the species, just like the Gibbs energy is additive in the chemical potentials of the species. The chemical potential $\mu_{i}$ of ion $i$, which is given by

$$
\mu_{i}=\mu_{i}^{\prime}+F z_{i} \phi_{i}
$$

has the same value in each phase at equilibrium, as can be shown by use of equation 5 . This constancy of $\mu_{i}$ at equilibrium throughout a multi-phase system is one of the most important ideas of chemical thermodynamics. At constant $T$ and $P, \mu_{i}$ is independent of the phase at equilibrium, even if the phases are different states of matter, are at different pressures, or have different electric potentials. Thus at equilibrium the transformed chemical potential $\mu_{i}^{\prime}$ has different values in phases with different electric potentials.

To find out more about the transformed chemical potential $\mu_{i}^{\prime}$, consider the fundamental equation for the transformed Gibbs energy $G^{\prime}$ for a multi-phase system with phases at different electric potentials. The differential of $G^{\prime}$ (defined by the Legendre transform in equation 50 ) is given by

$$
\mathrm{d} G^{\prime}=\mathrm{d} G-F \sum_{i=1}^{N} \sum_{i} \phi_{i} \mathrm{~d} n_{i}-F \sum_{i=1}^{N} \sum_{i} n_{i} \mathrm{~d} \phi_{i}
$$

Substituting the expression for $\mathrm{d} G$ from equation 5 yields

$$
\mathrm{d} G^{\prime}=-S \mathrm{~d} T+V \mathrm{~d} P+\sum_{i=1}^{N} \mu_{i} \mathrm{~d}_{i}-F \sum_{i=1}^{N} z_{i} n_{i} \mathrm{~d} \phi_{i}
$$

This shows that the electric potentials of the phases are natural variables of the transformed Gibbs energy. It is evident from equation 55 that the transformed chemical potential $\mu_{i}^{\prime}$ is given by

$$
\left(\frac{\partial G^{\prime}}{\partial n_{i}}\right)_{T, P, n_{j}, \phi_{i}}=\mu_{i}^{\prime}
$$

where $j \neq i$. This can be compared with the definition of the chemical potential of a species, which is given by

$$
\left(\frac{\partial G}{\partial n_{i}}\right)_{T, P, n_{j}}=\mu_{i}
$$

where $j \neq i$. There are corresponding molar transformed entropies and molar transformed enthalpies. Equation 55 also yields new Maxwell equations, a Gibbs-Duhem equation, and a Gibbs-Helmholtz 
equation. In a multi-phase system, the amounts of species are not independent variables because of the phase distribution constraints, and this is discussed elsewhere (ref. 21)

The electric potentials of phases are not natural variables of the Gibbs energy, but the electric potential of the phase an ion is in can be brought into calculations with the Gibbs energy by defining the activity $a_{i}$ of a species in a system having electric potential differences between phases with (ref. 21)

$$
\mu_{i}=\mu_{i}^{\circ}+R T \ln a_{i}+F z_{i} \phi_{i}
$$

The definition of the activity is simply a means for replacing the chemical potential of a species with a property, the activity $a_{i}$, that is more closely related with its concentration or partial pressure. Since the chemical potential of an ion in a phase is affected by the electric potential of the phase, it is reasonable to bring the electric potential into the definition of the activity in such a system. This means that the standard state chemical potential $\mu_{i}{ }^{\circ}$ of an ion in a multi-phase system with electric potential differences between phases is the value of the chemical potential at unit activity and at a reference electric potential for the whole multi-phase system. Substituting equation 58 into equation 5 yields

$$
\mathrm{d} G=-S \mathrm{~d} T+V \mathrm{~d} P+\sum^{N}\left(\mu_{i}{ }^{\mathrm{o}}+R T \ln a_{i}+F z_{i} \phi_{i}\right) \mathrm{d} n_{i}
$$

Note that this equation does not give the derivative of the Gibbs energy with respect to the electric potentials of the phases because they have been introduced as constants involved in the description of the system and are not natural variables of $G$. Bailyn ${ }^{22}$ has pointed out that $\mathrm{d} \phi_{i}$ does not appear in the GibbsDuhem equation corresponding with equation 59.

Equation 59 can be used to derive the equilibrium expression for a reaction that involves species in two phases with the same solvent:

$$
\mathrm{A}^{z \mathrm{~A}}(\alpha)+\mathrm{B}^{z \mathrm{~B}}(\alpha)=\mathrm{C}^{z \mathrm{C}}(\beta)
$$

The equilibrium condition derived from equation 5 is

$$
\mu\left(\mathrm{A}^{z} \mathrm{~A}, \alpha\right)+\mu\left(\mathrm{B}^{z} \mathrm{~B}, \alpha\right)=\mu(\mathrm{C} z \mathrm{C}, \beta)
$$

Substituting equation 58 yields the following expression for the equilibrium constant:

$$
\begin{aligned}
K & =\frac{a(\mathrm{C} z \mathrm{C}, \beta)}{a\left(\mathrm{~A}^{z \mathrm{~A}}, \beta\right) a(\mathrm{~B} z \mathrm{~B}, \beta)} \\
& =K \exp \left[-F\left(z_{\mathrm{A}}+z_{\mathrm{B}}\right)\left(\phi_{\beta}-\phi_{\alpha}\right) / R T\right]
\end{aligned}
$$

where

$$
K^{\prime}=\exp \left\{-\left[\mu^{\mathrm{o}}\left(\mathrm{C}^{z} \mathrm{C}, \beta\right)-\mu^{\mathrm{o}}\left(\mathrm{A}^{z} \mathrm{~A}, \alpha\right)-\mu^{\mathrm{o}}\left(\mathrm{B}^{z \mathrm{~B}, \alpha)}\right)\right] R T\right\}
$$

is the value the equilibrium constant would have if there was no electric potential difference. Issues of natural variables, components, and electroneutrality are dealt with elsewhere (ref. 21).

Equation 53 differs from the IUPAC recommendation (ref. 23), which is

$$
\tilde{\mu}_{i}=\mu_{i}+F z_{i} \phi_{i}
$$

where $\tilde{\mu}_{i}$ is referred to as the electrochemical potential and $\mu_{i}$ is referred to as the chemical potential. There are two problems with the IUPAC recommendation of 1974: The first problem is that $\tilde{\mu}_{i}$ is the chemical potential $\mu_{i}$ defined by equation 57 . The more serious problem is that $\mu_{i}$ on the right side of equation 64 is not the chemical potential for species $i$; it is given by the derivative in equation 56 and at equilibrium has different values in phases with different electric potentials.

\section{DISCUSSION}

Since the number of possible thermodynamic potentials for a system is given by $2^{k}$, where $k$ is the number of conjugate pairs of variables in the fundamental equation, it is important to be systematic and think about nomenclature problems when non-PV work is involved.

When the chemical potential $\mu_{\mathrm{s}}$ of a species is specified in a chemical reaction system, the conjugate extensive variable is the amount $n_{\mathrm{s}}$ ' of the $\mathrm{s}$ component, rather than the amount $n_{\mathrm{S}}$ of the s species. The amounts of components are independent variables when there are chemical reactions at equilibrium or phase equilibria, but amounts of species are not. In order to make $\mu_{\mathrm{s}}$ a natural variable, it is necessary to use a Legendre transform to define a transformed Gibbs energy $G^{\prime}$ by using $G^{\prime}=G-\mu_{\mathrm{s}} n_{\mathrm{s}}{ }^{\prime}$. The 
maximum number of components that can be removed in this way is $C-1$. There are four expressions for the transformed chemical potential of species $i$ :

$\mu_{i}^{\prime}=\left(\frac{\partial U^{\prime}}{\partial n_{i}^{\prime}}\right)_{S, V, n^{\prime} j \neq i, \mathrm{~s}, \mu_{\mathrm{S}}}=\left(\frac{\partial H^{\prime}}{\partial n_{i}^{\prime}}\right)_{S, P, n_{j}^{\prime} \neq i, \mathrm{~s}, \mu_{\mathrm{S}}}=\left(\frac{\partial A^{\prime}}{\partial n_{i}^{\prime}}\right)_{T, V, n_{j}^{\prime} \neq i, \mathrm{~s}, \mu_{\mathrm{S}}}=\left(\frac{\partial G^{\prime}}{\partial n_{i}^{\prime}}\right)_{T, P, n_{j} \neq i, \mathrm{~s}, \mu_{\mathrm{S}}}$

where $n^{\prime} j \neq i, \mathrm{~s}$ indicates that the amounts of all pseudoisomer groups except $i$ and $\mathrm{s}$ are held constant. There are corresponding molar transformed entropies and molar transformed enthalpies.

When a system involves work in addition to $P V$ work and chemical work, there at two possibilities, either the extensive properties in the additional terms are independent of $\left\{n_{i}\right\}$ or dependent on $\left\{n_{i}\right\}$. If the new extensive property is independent of $\left\{n_{i}\right\}$, it is a natural variable for $U, H, A$, and $G$. In this case a Legendre transform can be used to introduce the conjugate intensive property as a natural variable and the new thermodynamic potential can be designated with a new capital roman letter, following the custom of using $H, A$, and $G$. When Legendre transforms are used to define additional thermodynamic potentials by replacing some of the extensive natural variables $\{X\}$ with the corresponding intensive properties $\{P\}$, the chemical potential is given by

$$
\mu_{i}=\left(\frac{\partial \text { therm. pot. }}{\partial n_{i}}\right)_{\{P\}, n_{j \neq i},\{X\}}
$$

There are corresponding molar entropies and enthalpies.

If the new extensive property is dependent on $\left\{n_{i}\right\}$, as for a multi-phase system with phases at different electric potentials, the presence of this new work term does not affect the fundamental equations for $U, H$, $A$, and $G$. However, the introduction of the corresponding intensive variable as a natural variable using a Legendre transform leads to a transformed thermodynamic potential. The transformed thermodynamic potentials $U^{\prime}, H^{\prime}, A^{\prime}$, and $G^{\prime}$, which are very much like $U, H, A$, and $G$, yield new fundamental equations, Maxwell equations, Gibbs-Helmholtz equations, and a Gibbs-Duhem equation.

Acknowledgement. I am indebted to I. Oppenheim, G. N. Goldberg, F. J. Krambeck, and members of the Commission I.2 Committee on Legendre Transforms for many helpful discussions. I am indebted to NIH-1-RO1-GM48358-01A1 for support of this research.

\section{REFERENCES}

1. J. W. Gibbs. The Scientific Papers of J. Willard Gibbs, Vol. 1, Thermodynamics. Dover Publications, New York (1903).

2. H. B Callen. Thermodynamics and an Introduction to Thermostatics. Wiley, New York (1985).

3. W. R. Smith and R. W. Missen. Chemical Reaction Equilibrium Analysis. Wiley, New York (1982).

4. R. A. Alberty and I. Oppenheim. J. Chem. Phys. 89, 3689-3693 (1988).

5. R. A. Alberty and I. Oppenheim. J. Chem. Phys. 96, 9050-9054 (1992).

6. R. A. Alberty and I. Oppenheim. J. Chem. Phys. 98, 8900-8904 (1993).

7. J. A. Beattie and I. Oppenheim. Principles of Thermodynamics, Elsevier, New York (1979).

8. R. A. Alberty. I\&EC Fund. 22, 318-21 (1983).

9. R. A. Alberty, in F. J. Krambeck and A. M. Sapre (eds.). Chemical Reactions in Complex Systems, Van Nostrand Reinhold, New York (1991).

10. R. A. Alberty. Biophys. Chem. 42, 117-131 (1992).

11. R. A. Alberty. Biophys. Chem. 43, 239-254 (1992).

12. R. A. Alberty and R. N. Goldberg. Biochemistry 31, 10610-10615 (1992).

13. R. A. Alberty. Pure and Applied Chem. 65, 883-888 (1993).

14. R. A. Alberty. Biochem. Biophys. Acta 1207, 1-11 (1994).

15. R. A. Alberty and R. N. Goldberg. Biophys. Chem. 47, 213-223 (1993).

16. R. A. Alberty, A. Cornish-Bowden, Q. H. Gibson, R. N. Goldberg, G. G. Hammes, W. Jencks, K. F. Tipton, R. Veech, H. V. Westerhoff, and E. C. Webb. Pure Appl. Chem. 66, 1641-1666 (1994). (Also European J. Biochem., in press.)

17. R. A. Alberty. Biophys. J. 65, 1243-1254 (1993).

18. R. A. Alberty and F. J. Krambeck, in preparation.

19. R. A. Alberty. Chem. Rev. 94, 1457-1482 (1994).

20. R. A. Alberty. J. Electrochem. Soc. 142, 120-124 (1995).

21. R. A. Alberty and I. Oppenheim, in preparation.

22. M. Bailyn, A Survey of Thermodynamics, AIP, New York (1994).

23. R. Parsons. Pure Appl. Chem. 37, 499 (1974). 